\title{
Dificuldades encontradas pelos profissionais da saúde ao realizar diagnóstico precoce de autismo
}

\author{
Difficulties encountered by health professionals in the early diagnosis of autism
}

Céres de Oliveira Jendreieck

Pedagoga, especialista em Saúde Mental pela Universidade Positivo e Hospital Espírita de Psiquiatria Bom Retiro, acadêmica do curso de Psicologia da Universidade Federal do Paraná, professora da Rede Municipal de Educação de Curitiba, Curitiba, PR - Brasil, e-mail: ceres_oj@yahoo.com.br

Recebido: $26 / 04 / 2012$ Received: 04/26/2012

Aprovado: 18/11/2012 Approved: 11/18/2012

\section{Resumo}

0 presente artigo é resultado de uma pesquisa que teve como objetivo conhecer as dificuldades encontradas pelos profissionais da saúde ao realizar o diagnóstico precoce de autismo. Foram entrevistados oito profissionais da saúde responsáveis pelo diagnóstico ou que participam dele (pediatras, psiquiatras, psicólogos e neuropediatras). Foi utilizado um método qualitativo, de forma que não se buscou uma amostra representativa, mas sim a descoberta de indicadores dessas dificuldades de diagnóstico precoce presentes na prática clínica dos entrevistados. Os resultados das entrevistas foram organizados conforme os temas a que se referiam e depois discutidos. Esses resultados permitiram compreender que muitas das dificuldades apontadas podem ser enfrentadas por esses profissionais por meio de estudos aprofundados e atualização constante, práticas que possibilitem conhecer melhor o paciente e sua família, além de práticas interdisciplinares. 0 diagnóstico precoce e apropriado é importante para que se inicie o tratamento o mais cedo possível, com o objetivo de possibilitar melhores condições de vida para a criança autista.

Palavras-chave: Transtorno autístico. Pessoal de saúde. Diagnóstico precoce.

\begin{abstract}
This article is the result of a research that aimed to meet the difficulties found by health professionals when performing early diagnosis of autism. Eight health professionals responsible for or involved in the diagnosis (pediatricians, psychologists, psychiatrists and neuropediatricians) were interviewed. A qualitative method was used, since the goal was not obtaining a representative sample, but discovering indicators of these difficulties in the clinical practice of respondents. The results of the interviews were organized according to topics to which they referred and then discussed. These results allowed understanding that many of the difficulties pointed out may be faced by these professionals through deeper studying and constant updating, knowing the patient and his family better, and also through interdisciplinary practice. Early and appropriate diagnosis is important to start treatment as soon as possible in order to provide better living conditions for the autistic child.
\end{abstract}

Keywords: Autistic disorder. Health personnel. Early diagnosis. 


\section{Introduç̃̃o}

O presente artigo é resultado de uma pesquisa realizada com profissionais da saúde responsáveis pelo diagnóstico de autismo ou que participam dele. $\mathrm{O}$ objetivo desta pesquisa foi encontrar indicadores que apontassem para as principais dificuldades encontradas por esses profissionais ao realizar o diagnóstico precoce de autismo (antes dos três anos de idade).

Durante o estudo da bibliografia, percebeu-se que essas dificuldades provêm inicialmente da própria história do autismo. Diferentes estudiosos utilizaram o termo tanto para descrever sintomas de outros problemas mentais quanto para nomear síndromes diferentes. Esses problemas de definição geraram dificuldades para o processo de diagnóstico da doença. Hoje os instrumentos de diagnóstico, como o DSM-IV e a CID-10, proporcionam melhor clareza sobre a síndrome; entretanto, as dificuldades de diagnóstico permanecem, principalmente quando se trata de crianças com menos de três anos.

o termo autismo foi usado para descrever o isolamento social de adultos esquizofrênicos e, mais tarde, começou a ser usado por psiquiatras que estudavam crianças com comportamentos peculiares e com dificuldades em estabelecer relações sociais normais. Essa falta de consenso entre os pesquisadores resultou nas dificuldades em se estabelecer critérios de diagnóstico e testes de avaliação. Essas variedades de descrição geraram muitas discussões e transformaram o autismo num conceito amplo, chamado hoje de espectro autista, que engloba diferentes síndromes, e mesmo dentro dessas síndromes ocorrem variações de severidade (Wing, 1996).

Atualmente a síndrome do autismo não é, portanto, definida com precisão, e não há um acordo total sobre os testes permitindo medi-la (Leboyer, 1995).

As formas mais graves dos transtornos do espectro do autismo são diagnosticadas nos primeiros anos de vida, enquanto formas moderadas são identificadas apenas a partir da entrada na escola (Mandell, Novak \& Zubritsky apud Albores-Gallo et al., 2008).

A dificuldade de diagnóstico tem como consequência um atraso na busca dos recursos necessários ao atendimento e educação da criança. Consideramos que o diagnóstico precoce é importante porque propicia que a criança autista seja encaminhada o mais cedo possível para terapias e educação especializada, o que certamente resultará em melhores condições para seu desenvolvimento.

Wing (1996, p. 26) defende que "crianças com qualquer tipo de atraso de desenvolvimento ou perturbação necessitam educação e ajuda especial adequada ao seu problema ou problemas específicos". A importância da estimulação é também evidenciada por Brown (1996, p. 41) ao escrever que "quando o desenvolvimento espontâneo falha, o treinamento é a próxima e melhor alternativa. Qualquer que seja a extensão da incapacidade da criança, precisamos intervir precocemente no autismo, com um positivo programa, pessoal e planejado de atuação".

Compreendendo a importância do diagnóstico precoce surgiu a preocupação com a existência de dificuldades que possam atrapalhá-lo ou atrasá-lo.

De acordo com Albores-Gallo et al. (2008), é comum o diagnóstico errado. As famílias dessas crianças recebem frequentemente três ou quatro pré-diagnósticos. Isso gera custos elevados para os prestadores de serviços nas áreas de medicina e educação. Por várias razões os profissionais da saúde responsáveis pelo diagnóstico encontram numerosos obstáculos na avaliação. Evidenciar quais são essas dificuldades certamente ajudará na busca de recursos para enfrentá-las.

\section{Método}

A coleta de dados foi realizada através de entrevistas com profissionais da saúde responsáveis ou que participam do diagnóstico do autismo. Foram entrevistados oito profissionais, sendo três pediatras, dois psicólogos, um neuropediatra e dois psiquiatras. Foi utilizada uma entrevista semiestruturada, com questões abertas e fechadas. As entrevistas foram gravadas com a autorização dos participantes. A análise dos dados iniciou-se com a transcrição e leitura das entrevistas. A partir dessa leitura foram separados indicadores nas falas dos entrevistados. Esses indicadores foram selecionados pela repetição e organizados em temas para que fosse possível uma apresentação mais didática.

\section{Resultados}

Os resultados dessa pesquisa estão apresentados a seguir, a partir dos seis principais temas 
levantados nas entrevistas - os que se repetiram na fala de diferentes profissionais.

O primeiro tema foi "Encaminhamento". Esse tema refere-se a como as crianças com suspeita de autismo geralmente são encaminhadas aos profissionais da saúde entrevistados. Conforme as entrevistas, na maioria dos casos esse encaminhamento se dá pela própria mãe, pela escola ou por outros profissionais da saúde. As mães normalmente procuram o pediatra e este, quando suspeita de autismo, encaminha a criança para outros especialistas (neuropediatra, psiquiatra, psicólogo, fonoaudiólogo, etc.). Entretanto, algumas vezes a família não percebe os sintomas ou não procura ajuda e somente na escola o problema será notado e então encaminhado.

Sobre o segundo tema, "Procedimentos utilizados pelos profissionais para realizar o diagnóstico de autismo", os profissionais entrevistados citaram que no processo de diagnóstico de autismo utilizam a observação, a entrevista com os pais, a anamnese, a exclusão de outras doenças e os exames. De acordo com esses profissionais, o diagnóstico de autismo é um diagnóstico clínico, ou seja, depende da observação do comportamento da criança e de ouvir as queixas da família. É partindo dessa observação e dos relatos dos pais que se faz a anamnese.

0 diagnóstico de autismo é também um diagnóstico de exclusão, pois há necessidade de ter certeza de que os sintomas não estão sendo causados por algum outro problema. Por isso os profissionais entrevistados identificam a importância de solicitar avaliações oftalmológica e fonoaudiológica, além de exames como ressonância magnética, tomografia computadorizada e eletroencefalograma. 0 objetivo dessas avaliações e exames é descobrir se a criança tem problemas de visão ou audição e também verificar se há más-formações no cérebro ou convulsões associadas ao autismo.

Quanto ao terceiro tema, "Instrumentos que podem auxiliar o diagnóstico de autismo", foram citados pelos profissionais entrevistados jogos e brincadeiras, questionários e instrumentos de observação. Os entrevistados enfatizaram a importância de observar a criança enquanto ela brinca, por isso vários entre eles afirmaram ter brinquedos e jogos simples que são oferecidos à criança para que eles possam observar como ela reage quando é chamada para brincar e de que forma ela brinca; assim, esses profissionais podem verificar a presença ou ausência de alguns sintomas. Além disso, alguns dos profissionais entrevistados afirmaram que utilizam, quando necessário, instrumentos traduzidos de avaliação e observação.

Ao falar sobre o quarto tema, "Fatores que dificultam o diagnóstico precoce", os profissionais entrevistados apontaram para: 1) a dificuldade de conhecer melhor a criança e a família, uma vez que as consultas são muito curtas e o ambiente do consultório é muito diferente do ambiente ao qual a criança está acostumada; 2) a falta de maior conhecimento da família sobre o autismo; 3) a necessidade de observar a criança por mais tempo; 4) a falta de elementos importantes para o diagnóstico na entrevista com os pais; 5) a demora na realização dos exames; 6) a existência de diferentes graus de comprometimento dentro do espectro autista; 7) a necessidade do diagnóstico diferencial; 8) a necessidade de cuidado ao informar a família sobre o diagnóstico.

Sobre o quinto tema, "Sugestões para superar as dificuldades de diagnóstico", os profissionais entrevistados colocaram a importância de estudar mais sobre o autismo, procurar apressar os exames, solicitar aos pais que retornem brevemente para a próxima consulta e fazer que essas consultas sejam mais prolongadas, proporcionando uma observação mais cuidadosa e mais oportunidades para conhecer melhor a família e o paciente, fazer supervisão com profissionais mais experientes e fazer um trabalho interdisciplinar.

Quanto ao sexto tema, "Funções do diagnóstico precoce", os profissionais entrevistados falaram da importância de uma estimulação precoce do desenvolvimento cognitivo, afetivo e emocional da criança, assim como de sua fala. Outra função do diagnóstico precoce foi a de preparar a família para a "luta constante", conforme disseram os entrevistados. Quando há uma criança autista na família, há uma mudança em seu cotidiano. Isso inclui idas e vindas de diferentes médicos, laboratórios de exames, terapias etc., além de estarem o tempo todo atentos à criança autista e suas necessidades. Os entrevistados citaram também a importância do aconselhamento genético, ou seja, avisar a família que podem ocorrer outros casos de autismo nas futuras gerações e que as chances de recorrência de autismo entre irmãos são ainda maiores. Outra função do diagnóstico precoce, segundo os entrevistados, é possibilitar o encaminhamento para instituições 
especializadas no tratamento de autismo e orientar todos os profissionais que irão trabalhar com essa criança. Todas essas funções têm o objetivo de dar mais qualidade de vida para a criança autista e sua família.

\section{Discussão}

Os pais são geralmente as pessoas que têm um convívio mais intenso com a criança e por isso costumam ser os primeiros a perceber comportamentos estranhos e procurar um pediatra. Entretanto, há vezes em que os próprios pais demoram a perceber os sintomas e declaram que a criança teve um desenvolvimento normal nos primeiros anos de vida. O desenvolvimento motor geralmente se dá dentro do esperado e por isso as famílias demoram a perceber que há algo errado com a criança, o que só acontece quando percebem o atraso na fala. Essa questão é apontada por Campos (2005, p.21), quando afirma que "O desenvolvimento motor normalmente se processa dentro das etapas previstas, porém a criança é mais quieta e desinteressada em explorar o ambiente ao seu redor, bem como fixar o olhar e acompanhar as pessoas. Há extrema ansiedade com situações novas ou mudanças de ambiente".

Esse desinteresse pelo mundo ao redor e pelas pessoas muitas vezes passa despercebido pelos pais, que podem achar que é apenas uma característica própria da criança ou timidez, como relatou um dos profissionais entrevistados.

Mesmo entre pesquisadores há controvérsias quanto à idade do aparecimento dos sintomas. Leboyer (1995) explica que Kanner sugeriu o caráter inato do autismo desde a sua primeira publicação, mas anos depois Eisenberg e Kanner observaram que essa síndrome podia aparecer mais tarde.

Quando os pais não percebem "algo estranho" e não encaminham o filho para o pediatra, os professores percebem que há algum problema. Jordan (1996) aponta que algumas vezes os problemas de crianças autistas brilhantes não são aparentes até que elas estejam no ambiente social de uma escola. Nesse caso, alguns elementos importantes para o diagnóstico acabam faltando na entrevista realizada pelos profissionais, como foi apontado pelos entrevistados.

Depois que se percebe que há algo de diferente no desenvolvimento da criança e que surge a suspeita de que a criança tenha alguma síndrome do grupo dos transtornos invasivos do desenvolvimento, as dificuldades perduram. Diferentes síndromes possuem muitas características semelhantes, havendo necessidade de um diagnóstico diferencial que exige muitos detalhes e para tanto é necessário conhecer bem a criança.

Conforme Costa e Nunesmaia (1998, p. 25), "o diagnóstico do autismo infantil é baseado principalmente no quadro clínico do paciente, não havendo ainda um marcador biológico que o caracterize". Isso significa que não existe um exame que comprove se é autismo ou não, por isso são tão importantes o tempo para observação da criança, a conversa com os pais, a busca de informações na escola, a supervisão com profissionais mais experientes e o trabalho interdisciplinar.

Além disso, uma criança pode ter sintomas mais ou menos severos que outra, mesmo estando dentro de uma mesma categoria. Por exemplo, uma das áreas do desenvolvimento prejudicadas pelo autismo é a da comunicação; logo, a fala estará prejudicada. Há crianças autistas com a linguagem tão comprometida que não falam nenhuma palavra enquanto outras, mesmo com alguma dificuldade, conseguem falar e até mesmo ler e escrever.

Não é típico do desenvolvimento da fala seu surgimento antes dos dois anos de idade. Entretanto, uma inabilidade na comunicação certamente poderá ser notada desde cedo, pois mesmo antes de falar as crianças normais se comunicam utilizando outros meios (choro, sorrisos, gestos, olhares, brincadeiras).

Uma opção para buscar lidar com a dificuldade do diagnóstico precoce seria que os pediatras orientassem os pais para fazerem observações sobre a comunicação da criança em casa.

Principalmente quando se trata de autismo, o olhar do profissional para a família também é muito importante. Como os profissionais entrevistados declararam, há famílias que se desestruturam quando recebem o diagnóstico. Uma família desestruturada dificilmente contribuirá para o tratamento da criança autista e o profissional tem que estar consciente disso. Sprovieri (2005) aponta para os problemas enfrentados pelas famílias dos autistas e afirma que muitas vezes ter uma criança com transtorno invasivo do desenvolvimento significa para a família um luto, pois morre a fantasia daquela criança saudável, cheia de energia e perfeita com a qual 
os pais sonharam. Pais emocionalmente perturbados não poderão dar o apoio necessário a seus filhos, por isso o trabalho dos profissionais acaba se estendendo à família.

0 trabalho do profissional que busca realizar o diagnóstico precoce também pode ser facilitado conhecendo melhor o paciente e sua família, observando ambos dentro do consultório e fora dele. Visitar a escola da criança e conversar com seus professores permitirá que mais informações possam ser coletadas para a anamnese do paciente.

Percebendo o quanto é complexo para o profissional realizar o diagnóstico, direcionar o tratamento, orientar a família, percebe-se que o trabalho interdisciplinar é muito importante. Grillo e Silva (2004, p.37) apontam para esse aspecto quando dizem que "nenhum modelo teórico, sozinho, explica de forma abrangente e satisfatória a complexidade desta síndrome". Diferentes áreas do conhecimento contribuem para uma melhor compreensão sobre o transtorno autístico e seu tratamento. 0 diálogo, a troca de experiências e o trabalho conjunto poderão proporcionar a ampliação do conhecimento dos profissionais que dele participam e um trabalho mais completo que auxiliará o indivíduo autista nas suas diferentes necessidades, possibilitando a ele melhorar sua qualidade de vida.

\section{Considerações finais}

O correto diagnóstico precoce de autismo é importante para o planejamento, a previsão e utilização de serviços médicos e educacionais e a escolha de programas de intervenção e aconselhamento (Albores-Gallo et al., 2008).

Os resultados desta pesquisa permitiram compreender que muitas das dificuldades apontadas pelos profissionais da saúde entrevistados podem ser enfrentadas por meio de estudos aprofundados e atualização constante. A experiência é também um fator de grande importância, pois o profissional não pode prender-se a descrições. Cada criança tem suas peculiaridades, sua individualidade, sua história, e nenhuma corresponderá exatamente às descrições dos livros e manuais.

De acordo com Charman e Baird (apud AlboresGallo et al., 2008) as classificações do DSM-IV e CID10 não são suficientes para enfatizar a mudança na expressão dos sintomas ao longo do desenvolvimento. Eles descrevem apenas os critérios apresentados para a idade de quatro ou cinco anos, tornando-se difícil reconhecer o autismo em crianças entre 18 meses e três anos.

Por isso, considera-se que práticas que possibilitem conhecer melhor o paciente e sua família, como consultas mais prolongadas e frequentes, conversas com os professores e também as práticas interdisciplinares e supervisão com seus colegas mais experientes nesses transtornos, poderão auxiliar muito no trabalho.

As questões que foram explicitadas e discutidas a partir desta pesquisa não se esgotam aqui. Pelo contrário, o tema surgiu a partir da prática cotidiana dos profissionais, das diferentes posições teóricas e das realidades das crianças com transtornos invasivos do desenvolvimento, portanto essas questões permanecem abertas para novas reflexões sobre o diagnóstico de autismo.

\section{Referências}

Albores-Gallo, L. A., Guzmán, L. H., Pichardo, J. A. D. \& Hernández, B. C. (2008). Dificultades en la evaluación y diagnóstico del autismo: una discusión. Salud Ment, 31(1), 37-44. Recuperado em 20 de março de 2012, de http://www.scielo.org.mx

American Psychiatric Association. (1995). Manual diagnóstico e estatístico de transtornos mentais (DSM-IV). Porto Alegre: Artes Médicas. Recuperado em , de http://virtualpsy.locaweb.com.br

Brown W. (1996). Os primeiros anos. In K. Ellis (Org.), Autismo (pp. 29-56). Rio de Janeiro: Revinter.

Campos R. C. (2005). Aspectos neurológicos do autismo infantil. In W. Camargos Jr. (Org.), Transtornos Invasivos do Desenvolvimento: 3ํo Milênio (2a ed., pp. 21-23). Brasília: Presidência da República, Secretaria Especial dos Direitos Humanos, Coordenadoria Nacional para Integração da Pessoa Portadora de Deficiência.

Costa, M. F. \& Nunesmaia, H. G. S. (1998). Diagnóstico genético e clínico do autismo infantil. Arquivos de Neuropsiquiatria, 56(1), 24-31. Recuperado em 10 de março de 2012, de http://www.scielo.br 
Grillo, E. \& Silva, R. J. M. (2004). Manifestações precoces dos transtornos do comportamento na criança e no adolescente. Jornal de Pediatria, 80(2), 21-27. Recuperado em 06 de abril de 2009, de http://www. scielo.br

Jordan, R. (1996). Entre os seis e os doze anos de idade. In K. Ellis (Org.), Autismo (pp. 57-88). Rio de Janeiro: Revinter.

Leboyer, M. (1995) Diagnóstico e definição de autismo infantil precoce. In Autismo infantil: fatos e modelos (2a ed, pp. 9-37). Campinas: Papirus.
Organização Mundial da Saúde. (1997). Classificação Estatística Internacional de Doenças e Problemas Relacionados à Saúde (CID-10, 10a rev.). São Paulo: Universidade de São Paulo. Recuperado em ,de http://www.datasus.gov.br

Sprovieri, M. H. S. (2005). Aspectos familiares do portador de transtornos invasivos do desenvolvimento. In W. Camargos Jr. (Org.), Transtornos Invasivos do Desenvolvimento: 3o Milênio (pp. 99-106). Brasília: Presidência da República, Secretaria Especial dos Direitos Humanos, Coordenadoria Nacional para Integração da Pessoa Portadora de Deficiência.

Wing, L. (1996). Que é autismo? In K. Ellis (Org.), Autismo (pp. 1-27). Rio de Janeiro: Revinter. 